\title{
CGI scripts: Gateways to World-Wide Web power
}

\author{
JAMES M. KIELEY \\ Miyazaki International College, Miyazaki, Japan
}

\begin{abstract}
The power of the hypertext-based information presentation system known as the World-Wide Web can be enhanced by scripts based on the common gateway interface (CGI) protocol. CGI scripts residing on a Web server permit the execution of computer programs that can perform a wide variety of functions that may be useful to psychologists. Example applications are presented here, along with reference information for potential script developers.
\end{abstract}

The majority of information that people access via the hypertext-based information presentation system known as the World-Wide Web (WWW) is actually stored in the form of static files - that is, text and graphics files that appear a certain way when viewed from a Web browser, such as Netscape or Mosaic, because of a command language known as HTML. HTML, by its original design, is a simple command set used to present multimedia information that can be accessed asynchronously. The capabilities of HTML, and, therefore, the WWW, can be improved with scripts conforming to the common gateway interface (CGI) protocol. CGI, in effect, extends the WWW's presentation language (HTML) by permitting the execution of external programs from a WWW server. In addition to providing an overview of basic CGI concepts, this paper will present example applications that may be of interest to psychologists as well as pointers on how to get started in developing custom CGI scripts to fit a specific application.

Among the types of added WWW features that CGI scripts support are clickable area-sensitive graphics, known as imagemaps, that can be used in lieu of standard text menus, HTML FORMS that permit user-inputted data to be stored and/or analyzed, rotatable 3-D imaging, access to WAIS and SQL databases, and the building of indexes of selected WWW-based information. In theory, CGI gateway scripting permits programmers to add virtually any functionality that the original Web server software authors omitted.

\section{Essential Concepts and Vocabulary}

HTML stands for hypertext markup language, which represents the essential command set for formatting the presentation of Web documents. HTML commands are implemented as tags and are usually found in files ending with a .html extension (e.g., homepage.html). The original HTML 1.0 feature set has been superseded by HTML 2.0, which includes support for FORMS, and HTML 3.0, which supports tables, mathematical equations, and more flexible text formatting. HTML 2.0 and later FORMS in effect

Correspondence should be addressed to J. M. Kieley, The Claremont Graduate School, Academic Computing Building, 130 E. 9 th St., Claremont, CA 91711 (e-mail: jim@cgs.edu). permit users to input data by clicking on checkboxes, radio buttons, menus, reset buttons, and submit buttons, and also by typing into text fields (Lemay, 1995).

CGI was developed by the original programmers of the UNIX-based CERN and NCSA HTTP Web servers to supersede a prior scripting environment called HTBIN. Other Web servers that support scripting, including those based on other operating systems, may or may not use the CGI protocol. Early applications of CGI scripts included using them to serve information to a browser that is in a format that is otherwise unreadable, such as an SQL database.

An introduction to $\mathrm{CGI}$ is available from the National Center for Supercomputing Applications (see NCSA, 1995a). Netscape Communications (1995) publishes Web server documentation in hard copy form that may be valuable to CGI script writers. A general reference with a discussion of CGI worth obtaining is the book Managing Internet Information Resources (Liu, Peek, Jones, Buus, \& Nyre, 1994). Individuals interested in using CGI in conjunction with a Windows Web server are referred to Running a Perfect Web Site (Chandler, 1995).

HTML and CGI can work together by any of three alternative means. The ISINDEX tag provides almost any Web browser with the ability to use scripts to perform simple searches. Both the GET and the POST methods can be used to pass data input through HTML FORMS. The GET method passes user input using environmental variables, but GET messages have a 256 -character limit. The POST method passes keystrokes using standard input. The POST method does not have the limitations associated with ISINDEX and GET, and so its use is most preferred (see Liu et al., 1994, for a further discussion).

\section{Applications}

Since CGI provides WWW developers with an open development environment, there are few limits to potential applications. Presented here are five different uses that both are illustrative of what CGI scripts can do and may be of immediate benefit to psychologists in teaching or research. Exploration of these examples requires that the reader have full Internet access and a Web browser that supports FORMS. Examples can be tested interactively using the URL http://acb2.cgs.edu/psychpage.html. 
Source code for the CGI scripts is available at the same address.

1. Data collection. HTML FORMS provide compliant Web browsers with the user interface necessary to display newly inputted data as well as that which can be retrieved from a file. FORM capability alone is not enough to send and retrieve information. A program, usually implemented as a CGI script, is responsible for executing the actual data exchange. When data are sent by a gateway script, they can be written directly to a file or forwarded to another application, such as an e-mail program.

An example application of a gateway script that may be of interest to psychologists for research purposes is called psysurvey.cgi. This script supports the collection of survey data over the Internet. It has been adapted from scripts developed at the Georgia Institute of Technology GVU Center (1995). It works in conjunction with an HTML file called psy_survey.html. The latter file utilizes FORM tags that produce the fields necessary to input responses.

The most critical line of code from the psy_survey.html file is <FORM METHOD="POST" ACTION="http:// acb2.cgs.edu/cgi-bin/psysurvey.cgi"> FORM METHOD $=$ "POST" specifies the method for submitting information to the gateway script. The command inPUT TYPE $=$ "submit" then creates a button that, when selected, passes data on to the CGI script specified with the FORM METHOD command. The psysurvey.cgi script parses the data sent by the submit command and then forwards it, along with designated variable labels, to a specified e-mail address. Each variable name-value pairing is sent as a separate line in an e-mail message. This script can be modified to pass data to a file or a structured database in a format that can directly be read by a statistical analysis program (for related materials, see NCSA, 1995b). Data collection methods similar to those discussed here could also be used to collect accuracy responses to visual or auditory experimental stimuli presented using standard HTML tags.

2. Sharing expertise. Recently, it has been estimated that there may be as many as 10,000 different special interest forums that can be accessed via USENET news (for a description of this service, see Kieley, 1993). Some of these forums are relatively local and inactive, whereas others generate hundreds of messages per day that are accessible across the Internet. As work habits become more technologically based, many people find themselves becoming increasingly dependent on USENET news for obtaining technical expertise and for other types of free information sharing. There are two fundamental shortcomings associated with USENET news the way it is normally accessed: (1) the information is transient, with posted articles typically expiring and being inaccessible after only a few days, and (2) the search mechanisms of most news readers are limited.

Usenet-Web addresses the two aforementioned limitations of USENET news. One script included in the distribution is responsible for archiving postings from selected newsgroups. The archived information is easily searchable from a Web browser using another script. These archive and search functions are potentially important for USENET-dependent researchers and teachers with focused technical interests and with limited free time for browsing newsgroups. Usenet-Web users can search newsgroup archives at irregular intervals without worrying about having missed a key posting critical to answering a particular question.

Successful implementation of Usenet-Web requires the skills of someone familiar with both WWW server and UNIX system administration. It also requires that a WWW server have the ability to mount the necessary news-related volumes from a local news server. Normally, file sharing is done using NFS (Network File System). Although NFS is normally thought of as a UNIX file sharing protocol, other operating systems can access NFS volumes with the proper utility software. Thus, it may be possible to implement Usenet-Web on non-UNIX Web systems that also support CGI scripts written in Perl.

One factor that needs to be considered before setting up Usenet-Web is the disk space requirements for the longterm archiving of specified newsgroups. Usenet-Web installations may need to be restricted to a few newsgroups. The amount of activity within the selected groups will, of course, affect total storage requirements. A moderately active group may generate on the order of $1-2 \mathrm{Mb}$ of data per month.

Usenet-Web can be accessed for demonstration purposes at http://acb2.cgs.edu/cgi-bin/fetch. This site includes an archive of comp.infosystems.www.authoring. cgi, which is a forum that focuses on many topics discussed in this paper. A keyword search can be restricted by year, month, or day of posting. A search returns the titles of all postings that meet specified criteria. A user can then click on a given title to view a posting.

Usenet-Web can be accessed from virtually any WWW browser that supports FORMS. Usenet-Web can also be extended to act as an archive for listservs, such as SCiPL (Ransdell \& Anderson, 1995), using a utility program called mail2news (Salz, 1992). Configuration information for Usenet-Web is included with the distribution (see Franz, 1995).

3. Electronic testing. The Internet in general and the WWW in particular hold much promise for the direction in which education will move over the next decade. Increasing demand for classes coupled with decreased funding, which impact on numbers of both faculty and facilities, has many rethinking the education process. Distance education is a term that has been used to capture any teaching that takes place outside of a traditional classroom context.

There are now a number of distance education projects that use the WWW to distribute course materials. Typically, students sitting in a remote classroom or at home interactively point and click their way through information that a professor has made network accessible. This may include assignments, lab times, lecture notes, and even full-text readings.

CGI scripts permit the extension of the WWW for other classroom needs. For example, Cunningham (1995) has 
provided an example of how the Web can be used for objective testing. The original distribution includes two scripts written in standard C: (1) qform, used to automatically generate a form-based HTML file from a simple ASCII text file the test maker writes, and (2) qscore, used to score and send the test result back to the test taker along with a corrected list of inappropriate responses. Kieley (1995) has provided a modification to the original qscore program that automatically e-mails results to a designated address.

The URL http://acb2.cgs.edu/qform/qformA.html provides an example of the type of simple ASCII format that can be used to generate a test. The URL http://acb2.cgs. edu/qform/qformB.html shows portions of the HTML file that result after processing the ASCII file with the qform program. The URL http://acb2.cgs.edu/qform/ qformC.html shows how this document would appear in a browser that supports FORMS. The qscore script is responsible for grading a test when the submit button is pushed. The URL http://acb2.cgs.edu/qform/qformD.html shows the type of scored result that can be sent back to the test taker or forwarded to the instructor for recording.

4. Electronic conferencing. The term chat seen in isolation is usually interpreted as an abbreviation for the internet relay chat (IRC) service that was first introduced several years ago. IRC is a client-server technology that permits a group of people located anywhere with Internet access to have typed conversations in real time. In essence, this is an extension of the UNIX talk facility that supports typed real-time exchanges between individuals located at two workstations.

Historically, IRC has been a popular activity for college students sitting at university-owned workstations idling away the late-night hours, having conversations on any of a number of topics. The client software for early IRC implementations required knowledge of a fairly cryptic command set.

There are now a number of Web-based chat programs that function in a manner fairly similar to the original UNIX-based IRC programs. They vary in terms of their complexity and features. Psychat accessible at http://acb2. cgs.edu/webchat/psychat.html is an implementation of a program called Webchat offered by the Internet Roundtable Society (1995).

Psychat allows multiple individuals to have a text-based virtual conference in real time using a simple Web browser interface. The typed messages of participants can be accompanied by links to a graphics image in gif format. Typically, participants will type a URL (uniform resource locater) that is a link to pictures of themselves. Psychat also permits participants to include HTML links to other sites in their messages.

In addition to real-time propagation and accompanying images, Webchat has other technical advantages over listservs and Usenet news. Any posting needs only to reside on a single server to be world-wide accessible. This conserves disk space. Also, all postings are generally permanently archived.
5. Electronic indexing. At the time this was written, by some estimates the amount of information accessible via the WWW was growing at the rate of $10 \%$ per week. The number of WWW servers, while unknown, is now likely in the hundreds of thousands, and the number of individual documents is likely in the tens of millions. A web of information encompassing the entire planet is a great thing, but it is fully usable only if the information on it can be selectively and efficiently retrieved. The obvious tools for finding information are indexes; on the WWW, they essentially come in three varieties. Spiders (also known as robots) are computer programs that traverse the Web using various algorithms that automatically build searchable indexes. Trees are hierarchically organized indexes that require human intervention and decision making. A third class of index is represented by automatic indexing programs that are intended to make searchable only the information located within a given directory structure on a local server.

The amount of information on a given server or in a given directory structure can vary a great deal. For one recent class, I decided to abandon traditional textbooks and make all class-related information WWW-accessible. Thus, all reading materials, handouts, assignments, class exercises, and quizzes were distributed via a Web server. All told, the materials consisting of many different kinds of documents took up over $7 \mathrm{Mb}$ of disk storage.

Rather than require students to manually search through the information hierarchy each time they wanted to look something up, I provided two indexes now accessible at $\mathrm{http}: / / \mathrm{acb} 2 . c g s . e d u /$ indexes.html. One index is simply a tree diagram of the directory structure. Documents can be individually selected from the tree and then searched using a browser's built-in search function. This method is tedious when numerous documents must be searched. The other index is automatically generated from all directory documents. Titles of all documents containing at least one occurrence of a given search string can be retrieved at once using a CGI script. Returned titles can then be individually selected for full display of the file. The indexing and search scripts are collectively referred to as ICE indexing (Neuss, 1995).

Automatically generated Web indexes have the advantages of being up-to-date and of being thoroughly searchable in a single pass. Sometimes, the amount of information generated with such a search method can be overwhelming, and not all available indexing scripts provide the user with the ability to use Boolean search strategies.

\section{Writing CGI Scripts}

The easiest way to write CGI scripts is to find an existing script that closely approximates a desired function. A script that can be used unaltered is always preferred; however, in many cases, minor changes may be necessary. There are a number of example scripts in the public domain that can be retrieved over the Internet. Several can be retrieved from NCSA (1995c). A list of locations with additional examples is included in Table 1 . The most cur- 
rent version of this list can be accessed interactively from http://acb2.cgs.edu/cgi.html.

Choice of the most suitable programming language for writing new scripts from scratch depends on a number of factors, including the operating system platform a server is running under, whether or not performance is a critical issue, and, perhaps most importantly, preexisting programming expertise. To date, UNIX-based servers are still the overwhelming choice for WWW sites serving more than a few simultaneous users. Gateway scripts can be written in any of a number of popular languages found on UNIX systems, including C, Perl (short for practical extraction and report language), and TCL. UNIX shell scripts can also be used as CGI scripts, although execution speed may be less than desired using this method. CGI scripts can also be implemented on Macintosh OS, Windows NT, and Windows WWW servers. Scripting information for non-UNIX-based servers is available on the WWW. Table 1 lists several relevant locations.

As personal and small departmental Web servers continue to proliferate, a variety of more user-friendly tools to support added functionality will emerge. Recent announcements suggest that these tools will be object-based and require minimal levels of programming proficiency. Whether the majority of new tools developed to support client-initiated program execution will conform to the current CGI protocol is open to question.

As it stands now, CGI scripting is the predominant method for executing programs from a Web browser, and Perl is the language of choice for authoring CGI scripts. Perl generally has speed advantages over TCL, and, for a novice, it is not as difficult to learn as C. Two O'Reilly books, Learning Perl (Schwartz, 1993) and Programming Perl (Wall \& Schwartz, 1990) and a book by Till (1994) can be used to learn the elements of Perl.

The majority of existing CGI scripts were written for Perl version 4. Some scripts will also run under the recently released Perl 5, which is object-oriented in nature. The Perl language is freely distributed. A thorough electronic index that provides Internet access to Perl binaries, scripts, documentation, and help for a number of platforms, including UNIX, VMS, Mac OS, Windows, and Windows NT, is available (Seymour, 1995).

\section{The Future of CGI}

The continuing widespread adoption of the WWW as an information collection and distribution mechanism dictates that both current protocols and current development methods must be either significantly enhanced or replaced. This trend will ultimately affect the current CGI protocol.

Recent developments at university and corporate research labs provide some indication of what to expect in the future. For example, Java is a new language environment developed by SUN Computer that has now been adopted by Netscape Communications, perhaps the most dominant commercial WWW software company. Java has been designed as a simple-to-use, object-oriented, Internet-friendly, multiplatform, development system. VRML (virtual reality markup language) has many features that are similar to Java and also holds promise as a tool to further accelerate the development of network information services.

Table 1

Internet-Accessible CGI Scripting Resources

\begin{tabular}{ll}
\hline \multicolumn{1}{c}{ URL Location } & \multicolumn{1}{c}{ Description } \\
\hline http://cy-mac.welc.cam.ac.uk/cgi.html & Applescript/Frontier CGI Tour \\
http://www.bio.cam.ac.uk/web/form.html & CGI Form Handling in Perl \\
http://www.best.com/ hedlund/cgi-faq/ & CGI Programmer's Reference \\
http://www.boutell.com/cgic/ & cgic: an ANSI C library for CGI Programming \\
http://www.webedge.com/CGIinC & CGI's in C (Mac) \\
http://www.clark.net/pub/jrinker/cgi.htm & CGI..Common Gateway Interface Index \\
http://cy-mac.welc.cam.ac.uk/cgi.html & CGI Tour \\
http://www.intergalact.com/hp/hp.html & CGI Tutorials On-Line \\
http://wsk.eit.com/wsk/dist/doc/libcgi/libcgi.html & EIT's CGI Library \\
http://macsolutions.interstate.net/fmprocgi.html & File Maker Pro CGI gateway \\
http://www.comvista.com/net/www/WwWDirectory.html & Invented Worlds Software \\
http://java.sun.com/ & Java Home Page \\
http://www.uwtc.washington.edu/computing/ & \\
www/mac/cgi.html & MacWWW-CGI Applications \\
http://www.afternet.com/ michaelm/old_public/cgi/ & Michael's Guide to CGI Programming \\
http://www.halcyon.com/mooncrow/cgi.htm & Mooncrow's CGI/PERL Source Page \\
http://hoohoo.ncsa.uiuc.edu/cgi/overview.html & NCSA CGI Overview \\
http://marquis.tiac.net/software/parse-cgi.html & PARSE CGI OSAX (Macintosh) \\
http://www.uni-frankfurt.de/ fp/tools/proccginput.html & Processing Form Data in Shell CGI Scripts \\
http://ruulst.let.ruu.nl:2000/tcl-cgi.html & TCL-CGI home page \\
http://www.hyperion.com/ koreth/uncgi.html & Un-CGI version 1.5 \\
http://www.umr.edu/ cgiwrap/ & Using CGI at UMR (CGIWrap) \\
http://www.creation.com/code.html & Virtual Creation's CGI Programmers Page \\
http://Web.Actwin.Com:80/Newtype/vr/vrml/index.htm & VRML Gateway \\
http://www.charm.net/ web/Vlib/Providers/CGI.html & Web Developer's Virtual Library: CGI \\
http://www.Stars.com/ & Web Developer's Virtual Library \\
http://www.achilles.net/ john/cgi-dos/ & Win-httpd CGI-DOS \\
http://akebono.stanford.edu/yahoo/Computers/ & \\
World_Wide_Web/CGI_Common_Gateway_Interface & Yahoo CGI Listings \\
\hline &
\end{tabular}


Java, VRML, or similar language products may at some point replace the current methods as the preferred way to initiate program execution from a Web browser, but this is unlikely to occur soon. Official Web standards are evolving slowly in part because of the number of international institutions and companies involved in Web development. While the Internet continues to evolve, CGI will continue to find its place and allow Web enhancements to meet specific application needs.

\section{Conclusions}

The current WWW represents a significant first step toward integrating a wide variety of electronic information resources. The standard Web programming language, HTML, does not work this magic alone. HTML is a simple presentation language. HTML must rely on CGI gateway scripts to achieve its full potential at present.

With the currently available tools, CGI script writing is not something everyone will want to attempt. It is important to recognize some of the ways in which standard WWW technology can be enhanced by programs ascribing to the CGI protocol. Existing CGI programs may be available to fit a given application unaltered. In other instances, minor modifications may be able to adapt a script for a specific use. Under some circumstances, scripts may have to be developed from scratch.

As commercial developers become more invested in the WWW, custom Web extensions will become easier to develop for nonprogrammers. It is too early to tell which methods will eventually become dominant, but it is clear that Web technology will evolve in the direction of extended functionality and more user-friendly control.

\section{REFERENCES}

CHANDLER, D. M. (1995). Running a perfect Web site. Indianapolis, IN: Que Corporation.
CunNingham, R. (1995). The aform package [On-line]. Available URL: http://www.satlab.hawaii.edu/space/hawaii/qform.html

FranZ, B. (1995). Usenet-Web 1.0.2 [On-line]. Available URL: http: //www.netimages.com/ snowhare/utilities/usenet-web/

Georgia Institute of Technology GVU Center (1995). GVU's WWW user survey home page [On-line]. Available URL: http://www. cc.gatech.edu/gvu/user_surveys/

INTERNET ROUNDTABLE SOCIETY (1995). Webchat. [On-line]. Available URL: http://www.irsociety.com/webchat.html

KIELEY, J. M. (1993). Integrating remotely accessible information services into psychology instruction and research. Behavior Research Methods, Instruments, \& Computers, 25, 287-294.

KIELEY, J. M. (1995). Qscore modifications [On-line]. Available URL: http:// acb2.cgs.edu/qscoremod.html

LEMAY, L. (1995). Teach yourself Web publishing with HTML in a week. Indianapolis, IN: Sams.

Liu, C., Peek, J., Jones, R., Buus, B., \& Nyre, A. (1994). Managing internet information resources. Sebastopol, CA: O'Reilly.

NCSA (1995a). The generic SQL gateway to the World-Wide Web [Online]. Available URL: http:/hoohoo.ncsa.uiuc.edu/CGI/overview.html

NCSA (1995b). The generic SQL gateway to the World-Wide Web [Online]. Available URL: http://fiaker.ncsa.uiuc.edu:7999/gsqlbof/

NCSA (1995c). The generic SQL gateway to the World-Wide Web [On-line]. Available URL: ftp://ftp.ncsa.uiuc.edu/Web/httpd/Unix/ ncsa_httpd/cgi/

NetSCAPE CoMmuniCATIONS (1995). Netscape communications home page [On-line]. Available URL: http://home.netscape.com/

NEUSS, C. (1995). Ice indexing server extension for $W W W$ servers [Online]. Available URL: http:// www.ham.muohio.edu/./ICE

RANSDELL, S. E., \& ANDERSON, M. D. (1995). Establishing a SCiP list for year-round discussion: Keeping the information ball rolling. $\mathrm{Be}$ havior Research Methods, Instruments, \& Computers, 27, 116-119.

SALZ, R. (1992), mail2news [On-line]. Available URL: ftp://ftpmail/ftp. elelab.nsc.co.jp/pub/net/news/mail2news.tar.gz

Schwartz, R. L. (1993). Learning Perl. Sebastopol, CA: O'Reilly. SEYMOUR, R. (1995). Usenet-Web 1.0.2 [On-line]. Available URL: http://www.ee.pdx.edu/ rseymour/perl/

TILL, D. (1994). Teach yourself Perl in 21 days. Indianapolis, IN: Sams.

Wall, L., \& SchWartz, R. L. (1990). Programming Perl. Sebastopol, CA: O'Reilly.

(Manuscript received November 13, 1995; revision accepted for publication February 12, 1996.) 